\title{
The Privacy of the Academic Community in Mapping Usage Patterns over Wi-Fi Connections
}

XXIV International Conference of the Iberoamerican Society

of Digital Graphics Medellin | Colombia

\author{
Luís Henrique Pavan \\ Universidade Federal de Santa Catarina | Brasil | luis.henrique.pavan@posgrad.ufsc.br \\ Lucas Fernandes de Oliveira \\ Universidade Federal de Santa Catarina | Brasil | fernandes.lucas@grad.ufsc.br \\ Gabriel Machado da Rosa \\ Universidade Federal de Santa Catarina | Brasil | gabriel.m.rosa@grad.ufsc.br \\ José Ripper Kós \\ Universidade Federal de Santa Catarina | Brasil | jose.kos@ufsc.br
}

\begin{abstract}
Data sourced by mobile devices has gained importance in urban research techniques. We have conducted two different studies on the usage pattern of a university campus using Wi-Fi connections. We have evaluated this data using anonymization methods more restricted than the Brazilian Data Protection Law. One of our studies is an ongoing work on the campus reopening after the coronavirus crisis. We sought to obtain data that may constitute resources to resilient urban projects on campus. Our results highlight the liability of the more restricted anonymization method and the quality maintenance of the data representation simultaneously.

Keywords: Wi-Fi connections; Data protection; Data visualization; University campus; Social infrastructure
\end{abstract}

\section{INTRODUÇÃO}

As ferramentas de trabalho dos profissionais de arquitetura e urbanismo têm mudado significativamente nas últimas décadas. Alguns pesquisadores acreditam que hoje as cidades estão mais "projetáveis" do que já estiveram antes. Ao mesmo tempo, a capacidade atual em digitalizar, analisar, manipular e prever os resultados de edifícios, infraestruturas e sistemas urbanos ampliou o alcance e o impacto das decisões dos administradores e projetistas (Yang \& Yamagata, 2020). Mecanismos de Sistemas Sociotécnicos, como os telefones celulares e suas redes, representam um novo tipo de infraestrutura capaz de colaborar sensivelmente no planejamento dos espaços públicos urbanos.

Neste trabalho, realizamos dois estudos nos quais coletamos dados de Wi-Fi para demonstrar diferentes dinâmicas humanas no campus sede da Universidade Federal de Santa Catarina (UFSC). No primeiro estudo, os dados de trajetória demonstram a integração de alunos de diferentes centros de ensino, auxiliando na identificação de locais de confluência social dentro do campus. O segundo estudo trata da avaliação da densidade dos edifícios através do uso da rede Wi-Fi. Este último se encontra em desenvolvimento no contexto de planejamento de retomada das atividades no campus, interrompidas em março de 2020 devido ao novo coronavírus, dispensando metodologias de pesquisa mais custosas ou que dependam do acompanhamento humano, seja através de entrevistas ou de contagem, para levantamento dos dados.
Para representação e visualização dos dados, optamos por utilizar a aplicação Kepler.gl. Um aspecto importante do aplicativo utilizado para visualização dos dados é a facilidade na execução dos gráficos. O Kepler.gl apresenta velocidade de processamento dos dados superior a softwares de cartografia tradicionais, facilitando consultas e visualizações. Esses dados corroboram na identificação de locais em que os cuidados em uma retomada parcial das atividades, prevista pela universidade como uma etapa de transição (UFSC, 2020), deverão ser observados cautelosamente e com atenção não somente às salas de aula, mas as demais dependências físicas, como espaços de circulação e banheiros, por exemplo.

O território universitário é um local de alto risco por inúmeros fatores. Sobretudo em salas de aula e demais espaços fechados, a manutenção do distanciamento social encontra sérias dificuldades. Procuramos iniciar essa discussão sobretudo porque os planos da universidade incluem essa retomada gradual e estudos da natureza que propomos podem ser uma maneira viável de reorganização institucional no período de readaptação do território físico da universidade na retomada presencial de atividades.

Uma questão crucial que tangencia a abordagem proposta é a da interdependência existente entre a privacidade dos usuários e o processo de formulação dos algoritmos responsáveis pela automatização dos raciocínios, que nos permitem trabalhar com os dados obtidos. Os dois estudos apresentados problematizam essa questão em face da Lei Geral de Proteção de Dados Pessoais (LGPD - Lei 
13.709/18) e da multidisciplinaridade envolvida na formulação dos algoritmos utilizados. Destacamos o potencial destes dados na identificação de dinâmicas humanas no campus com métodos de anonimização mais restritivos que a LGPD, garantindo maior segurança aos usuários. Partimos da avaliação dos dados obtidos e sua visualização, bem como descrevemos os desafios entre se obter o dado de qualidade para a pesquisa em ambientes interdisciplinares e as adequações à legislação.

Fundado em 1960, o campus sede da UFSC localiza-se na cidade de Florianópolis, região sul do Brasil. Trata-se de um campus urbano, circundado por cinco bairros cuja população estimada pela prefeitura em 2015 era de aproximadamente 80 mil habitantes. O primeiro projeto territorial para o campus data de finais da década de 1950 e, apesar de não ter sido implantado integralmente, é um importante determinante, já que estabeleceu o traçado viário da sede da universidade. Destaca-se a existência de uma microbacia hidrográfica com aproximadamente $4,5 \mathrm{~km}^{2}$ no campus. A setorização do campus em centros de ensino e departamentos é acentuada pela presença dos córregos que hoje, sobretudo devido ao uso e ocupação de suas margens por estacionamentos, acentua a segmentação territorial existente.

\section{METODOLOGIA}

Dados de trajetória são sujeitos a ataques mesmo após serem anonimizados. O algoritmo que utilizamos na anonimização de dados foi construído em parceria com o Laboratório de Segurança da Informática e a Superintendência de Governança Eletrônica e Tecnologia da Informação e Comunicação, ambos da UFSC. Durante um dia típico, mais de 1,5 milhões de registros de conexão (contabilizando mais de uma conexão do mesmo dispositivo em diferentes momentos) são armazenados no sistema da universidade. Eles incluem informações como coordenadas dos pontos de acesso, que serão utilizados aqui como pontos de um trajeto, e o número de matrícula, que permite associar outras informações relacionadas ao cadastro do usuário, enriquecendo os dados com informação contextual.

A anonimização acontece a partir de um método chamado de Mix $\beta$-k-anonymity, no qual um quase-identificador pessoal é utilizado no agrupamento de pessoas e suas trajetórias. Tanto $\beta$ quanto $\mathrm{k}$ são parâmetros que orientam o nível de privacidade que se deseja aplicar ao dado. $\mathrm{Na}$ prática, o método considera um $\mathrm{k}$ de pelo menos cinco estudantes vinculados ao mesmo centro de ensino, significando que qualquer grupo menor do que cinco acessando o mesmo roteador no mesmo intervalo de tempo será desconsiderado na coleta de dados, garantindo assim maior privacidade aos grupos. A opção por um $\mathrm{k}$ equivalente a cinco, bem como suas implicações técnicas na coleta e base de dados estão conforme Mangrich et al., (2019).

Com a finalidade de aumentar a segurança dos dados pessoais e a qualidade do dado obtido é aplicado o conceito de trajetória semântica. Diferente do conceito tradicional de trajetória, que somente descreve uma sequência restrita de pontos no espaço com uma informação temporal, a trajetória semântica é baseada no conceito de stops and moves. As paradas (stops) são determinadas por um período mínimo de permanência em locais de interesse, enquanto os deslocamentos (moves) são determinados pela diferença de tempo e distância entre pontos de interesse consecutivos, onde cada evento pode receber uma informação contextual. Além disso, os pontos de acessos são agrupados em áreas de interesse maiores, a exemplo do agrupamento de todos os pontos de roteadores de uma biblioteca em um único ponto, atribuindo uma informação semântica como "biblioteca" (stop), generalizando os ambientes e ocultando a posição exata das conexões dentro do edifício. $\mathrm{O}$ agrupamento permite também que grupos de 5 pessoas $(k=<5)$ que não seriam contabilizadas pelo algoritmo, passem a ser incluídas como parte de um grupo maior de pessoas, garantindo a qualidade do dado e a segurança dos usuários (Mangrich et al., 2019). Destacamos que nossa postura de anonimização visa aperfeiçoar alternativas que trabalhos semelhantes podem seguir frente às leis de proteção de dados.

O arquivo resultante de todo processo de anonimização é gerado em formato CSV e pode ser visualizado diretamente na ferramenta Kepler.gl, aplicação web para visualização de dados geoespaciais de larga escala e de alta performance. Diferente dos softwares de cartografia utilizados habitualmente, o Kepler.gl é uma ferramenta open source que apresenta uma interface bastante amigável ao usuário não especializado, com o objetivo explorar grandes volumes de dados de forma rápida e interativa, se tornando uma ferramenta de aprendizagem e pesquisa para os mais diversos campos de conhecimento. Com a mesma base de dados anonimizada, dois diferentes estudos foram realizados. A estrutura das tabelas de informações depende da necessidades de cada estudo.

\section{ESTUDO 1}

A amostra utilizada no estudo 1 é uma coleta de dados do dia 10/10/2019 que representa as trajetórias semânticas realizadas por estudantes de 4 centros de ensino diferentes, entre período das $6 \mathrm{~h}$ e $10 \mathrm{~h}$ da manhã, indicando locais de interesse onde ocorre o que chamamos de confluência social. Os dados são estruturados em um arquivo CSV (Tabela 1).

No kepler.gl, as trajetórias são visualizadas através do layer de linhas, que utiliza a latitude e a longitude do local de origem e destino para traçar uma reta. A espessura das linhas é determinada pela quantidade de pessoas no local de origem, ou seja, pessoas que estão se deslocando em direção ao destino (Figura 1). Para resolver a sobreposição de trajetórias, o kepler.gl disponibiliza uma opção de mistura de camadas chamada additive, criando um efeito visual de iluminação que destaca ocorrências sobre o mesmo ponto. Outro layer do tipo cluster foi criado para auxiliar na visualização da densidade populacional em cada local. (Figura 2) A escala de cores dos clusters utilizou como parâmetro o total de pessoas conectadas no

Tabela 1: Dados de composição do arquivo CSV.

$\begin{array}{lllllllllll}\begin{array}{l}\text { de } \\ \text { ensino }\end{array} & \text { origem } & \text { origem } & \text { origem } & \text { origem } & \text { P. org. } & \text { destino } & \text { destino } & \text { destino } & \text { destino } & \text { P. des } \\ \text { CCE } & \text { H3 } & 10 / 10 / 2019 & - & - & 6 & \text { A2 } & 10 / 10 / 2019 & - & - & 42 \\ & & 07: 00 & 27.600442 & 48.525172 & & & 07: 15 & 27.600836 & 48.519833 & \end{array}$



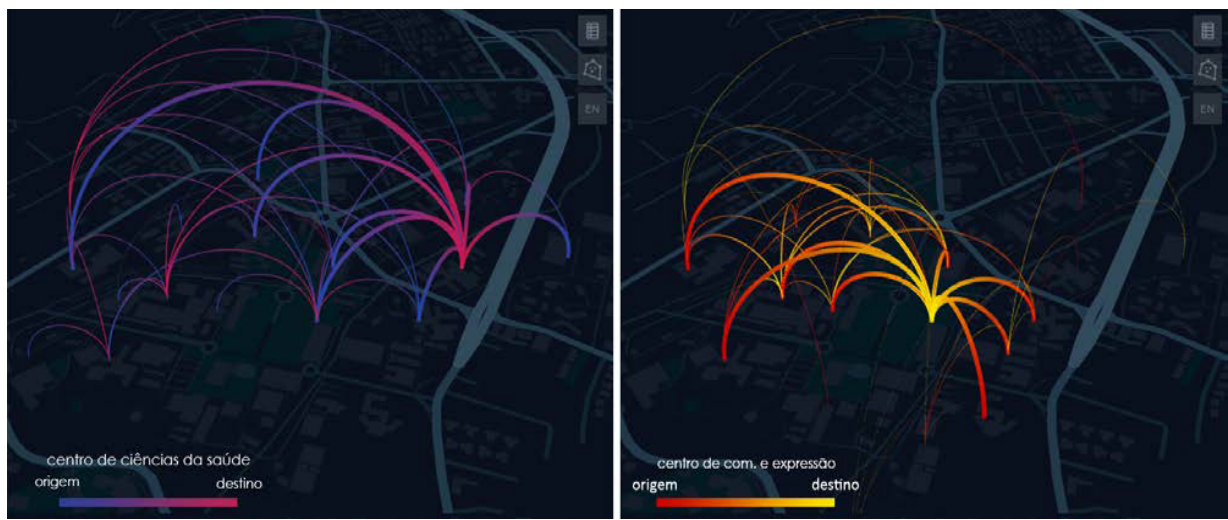

Figura 1: Trajetórias dos graduandos de diferentes centro de ensino, o centro de ciências da saúde e o centro de comunicação e expressão, às 8 horas. $O$ método de representação explora a visualização em 3D.

local de destino. Os clusters são agrupamentos de dados baseados em determinado raio geoespacial, isto é, todos os pontos dentro do raio determinado serão agrupados, tornando-se um único objeto, círculos com raios maiores indicam locais com um agrupamento maior de pontos de acessos. A coluna contendo o timestamp (formato data yyyy:mm:dd hh:mm) das conexões é utilizada como filtro de horário para o mapa, diferentes horários e intervalos de tempo podem ser explorados e animados através de uma interface representando a linha do tempo presente na coluna.

\section{ESTUDO 2}

No estudo 2 demonstramos outro exemplo de aplicação da base de dados de conexões obtidas através do log de usuários anonimizado. Neste caso, optamos por não utilizar o agrupamento de pontos de acesso, permitindo identificar com mais precisão a intensidade de uso em locais específicos do campus. Diferente do estudo 1 , utilizou-se a base completa de dados sem agrupamento por curso ou centro de ensino. Considerando somente a coordenada geográfica dos roteadores, obteve-se um mapa que representa a densidade de ocupação dos locais através dos layers heatmap e cluster. (Figura 3). Os roteadores utilizados no campus têm abrangência de aproximadamente 50 metros de raio e não são individualizados por salas de aula, tornando pouco acurada a aferição da densidade de pessoas por ambientes específicos. Contudo, aferir a densidade dos edifícios através dos roteadores nos permite auxiliar as comissões técnicas encarregadas das primeiras decisões a respeito da retomada de atividades no campus, identificando os pontos mais críticos.

A amostra contém os registros de um período de 24 horas do dia 10/10/2019 com um pico de 34.323 usuários conectados às $16 \mathrm{~h} 00$. Em ambos os layers, a densidade de cada local é determinada diretamente pela quantidade de pessoas conectadas no mesmo ponto de acesso. O raio das manchas de calor utiliza o mesmo raio de abrangência dos roteadores, 50 metros, demonstrando com mais precisão a intensidade de usos em pontos específicos. O layer cluster auxilia na contagem de pessoas, permitindo consultar a população em determinadas regiões através da seleção de pontos de acesso dentro de um determinado raio.

Essas imagens (Figura 3) constituem uma pesquisa em desenvolvimento. Incluí-las neste trabalho deu-se pela relevância e emergência do tema no contexto da avaliação

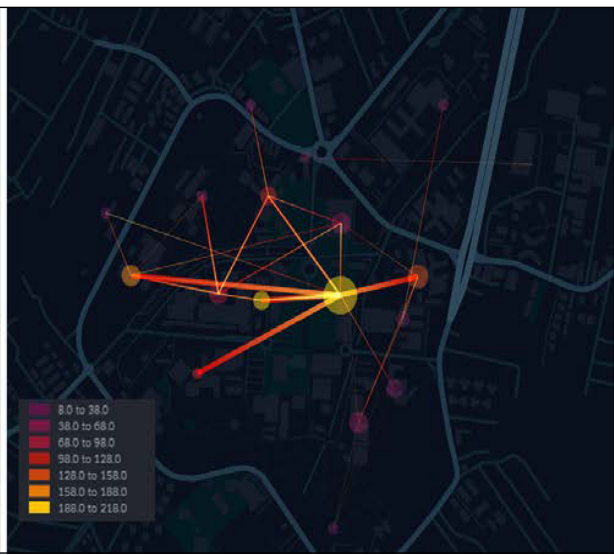

Figura 2: base de horário e intervalo constantes com representação ortogonal. A escala de cores do cluster utiliza por parâmetro a quantidade de pessoas no local de destino e círculos com raios maiores indicam locais com densidade maior de pontos de acesso 

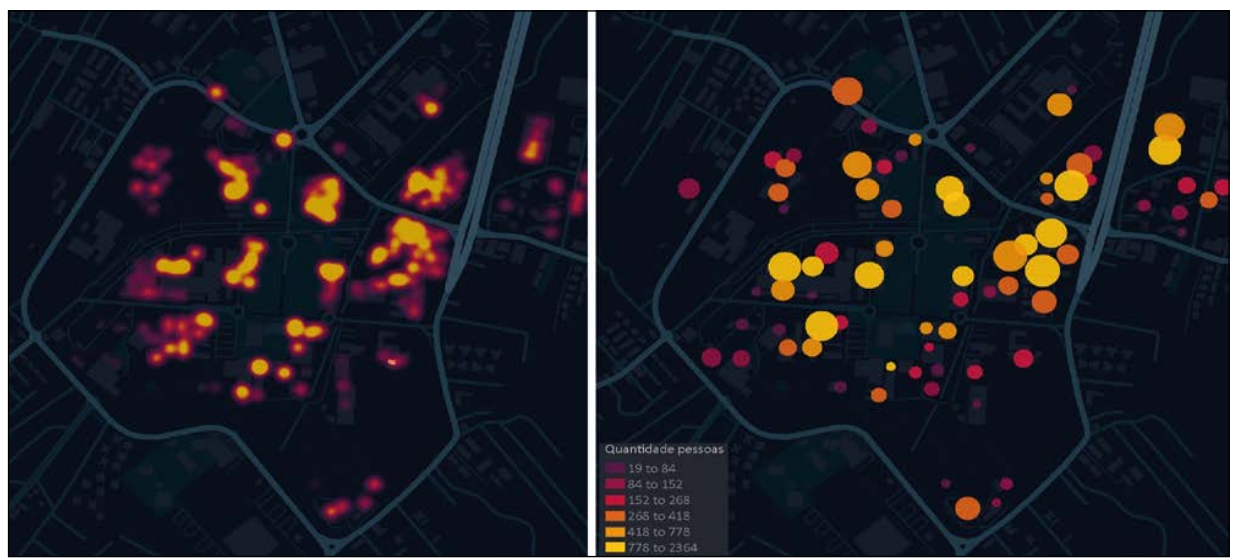

Figura 3: Mapa de calor e mapa de cluster gerados com a base de dados indicando locais com maiores concentrações de conexões

de retomada da ocupação do campus da UFSC após a crise sanitária causada pela pandemia do novo coronavírus (OMS, 2020-1) considerando a necessidade de distanciamento social imposta pelo SARS-CoV-2, causador da doença chamada COVID-19 (Yang et al., 2020). A Organização Mundial da Saúde (OMS) tem recomendado um distanciamento mínimo de um metro e meio por pessoa (OMS, 2020). O método proposto auxilia a universidade no controle de seus espaços físicos, já que ela não tem real conhecimento da quantidade de pessoas que utiliza diariamente suas dependências físicas, sendo a distribuição de espaços destinados à salas de aula um indicador que nem sempre corresponde à real dinâmica de uso do espaço físico do campus.

\section{RESULTADOS}

Nos resultados do estudo 1 foram obtidos os principais trajetos dos grupos de estudantes vinculados a determinados centros de ensino. Como a base de dados utilizada no Kepler.gl continha quatro centros de ensino localizados no campus, optamos por demonstrar neste trabalho apenas dois centros de ensino específicos, facilitando assim a compreensão mais imediata do objetivo do método.

Nas Figuras 1 e 2, são demonstrados os deslocamentos dos grupos de estudantes através das linhas, enquanto as cores indicam quais são os pontos mais frequentes de origem e destino desses grupos dentro do campus, representando a geoespacialização do arquivo anonimizado contendo as trajetórias realizadas por grupos de estudantes às $8 \mathrm{~h}$. As trajetórias permitem identificar quais porções territoriais do campus são menos frequentadas pelos graduandos de diferentes cursos.

No estudo 2, em ambos os mapas demonstramos os locais que devem ser considerados mais críticos em um cenário de retomada das atividades. Observa-se que a densidade é muito mais acentuada em alguns setores do campus do que em outros, correspondendo justamente aos diferentes centros de ensino (Figura 3).

\section{DISCUSSÃO E CONCLUSÃO}

\section{PRIVACIDADE, LGPD E A CONSTRUÇÃO DO ALGORITMO DOS ESTUDOS}

A Constituição Federal brasileira protege a intimidade dos brasileiros nos âmbitos da vida privada e da intimidade humana. (Brasil, 1988) Deve-se ter em mente que o conceito de privacidade é mutável, acompanhando aos valores da sociedade. Mulholland (2018) afirma que a conceituação de privacidade habitual tem sido superada. Segundo a autora, o direito à privacidade, tradicionalmente vinculado ao direito de ser deixado só, inclui cada vez mais a tutela de dados sensíveis, de seu controle pelo titular e, especialmente, respeitando a liberdade de escolha pessoal de caráter existencial.

Nesta seara, a proteção de dados pessoais é um requisito essencial da democracia. O tratamento de dados pode ter comportamento duplo, dada a crescente capacidade de processamentos cada vez mais precisos e abrangentes. 0 trabalho de dados em larga escala permite contemplar os interesses de pessoas ou entidades determinadas, melhorando políticas públicas, por exemplo, ao mesmo tempo em que pode retirar a capacidade de autonomia do indivíduo e o seu direito de acesso ao consumo de bens e serviços de interesse comum (Mulholland, 2918).

No Brasil, a LGPD em seu Art. $1^{\circ}$ dispõe sobre 0 tratamento de dados pessoais, inclusive nos meios digitais, com o objetivo de proteger os direitos fundamentais de liberdade e de privacidade e o livre desenvolvimento da personalidade da pessoa natural (Brasil, 2018). A LGPD tem em sua concepção uma influência marcante da Europe Union General Data Protection Regulation. Esta lei vigora desde 2018 no território da União Europeia (EU), incluindo organizações que visam o mercado interno da $E U$, independentemente do seu local de estabelecimento (Voigt, P., \& Von dem Bussche, A., 2017).

São excetuados da lei, através do Art. $4^{\circ}$, o tratamento de dados pessoais realizado por pessoa natural para fins exclusivamente particulares e não econômicos; realizado para fins exclusivamente jornalístico e artísticos; ou acadêmicos quando atendidas as exigências dos artigos $7^{\circ}$ e 11 , que versam, entre outros aspectos, sobre a 
realização de estudos por órgão de pesquisa, garantida, sempre que possível, a anonimização dos dados pessoais.

O desenvolvimento dos algoritmos capazes de obter os dados que necessitamos é um trabalho conjunto realizado entre áreas do conhecimento cada vez mais próximas. O conhecimento técnico dos arquitetos e urbanistas e dos profissionais de tecnologias da informação e comunicação. Acreditamos que a natureza multidisciplinar presente neste trabalho seja também um estímulo para uma integração mais efetivas entre áreas distintas do conhecimento.

\section{O CAMPUS, A CIDADE E AS FERRAMENTAS QUE APRESENTAMOS}

No estudo 1 nossa avaliação tomou em conta a configuração espacial, edilícia e funcional dessas localidades. Considerados a partir de uma visão ampliada de sua relação institucional e projetual, tais locais podem ser plausíveis para uma maior influência e participação na esfera urbana, municipal ou mesmo regional, facilitando atividades que reforcem o seu caráter de infraestrutura social. De modo geral, a configuração tipológica dos campi no Brasil segue uma lógica espacial guiada pela segmentação do território através dos diferentes centros de ensino que compõem a universidade. Os edifícios do campus da UFSC seguem uma lógica monofuncional cuja vinculação aos respectivos departamentos a acentua, desfavorecendo a integração entre acadêmicos de cursos distintos, por exemplo.

No campus da UFSC essa divisão é reforçada pelo tratamento dado aos cursos d'água que o permeiam. $\mathrm{Na}$ maioria das vezes, esses córregos estão associados a estacionamentos e, apesar da potencialidade axial que possuem, o tratamento do entorno desestimula o uso desses canais como orientadores dos caminhos. Por isso, conhecer e ser capaz de visualizar as dinâmicas de permanência e deslocamento da comunidade acadêmica nos permite demonstrar aos projetistas do campus quais os lugares e características de uso das centralidades e caminhos do campus, além de quais setores estão mais fragilizados em termos de passagem de pessoas. Os deslocamentos indicados podem justificar projetos que visem reforçar o papel dos córregos presentes no campus e criar oportunidades de bons locais para caminhadas. Os córregos do campus se conectam à cidade axialmente, tornando oportuna a conexão entre os cinco diferentes bairros da região universitária, utilizando rotas exclusivas de pedestres.

Ratti et al. (2006) relacionam a complexidade dos espaços públicos ao impacto das tecnologias móveis nos hábitos humanos, requerendo novas técnicas de pesquisa onde o uso destas informações é um componente essencial. Posto o grande volume de dados gerado pelas conexões no campus, o método apresentado demonstra grande potencial no reconhecimento das dinâmicas acadêmicas e nos padrões de uso do campus. Sabe-se que o tipo de pesquisa aqui apresentado é um campo em expansão que representa, dependendo da abordagem, uma gama de possibilidades ou uma série de riscos à privacidade e segurança dos usuários (Bratton, 2006). As dinâmicas identificadas são consideradas em uma visão ampliada de sua relação institucional e projetual. As análises podem ser relevantes para maior influência e participação nas esferas urbana, municipal e regional, facilitando atividades que reforcem o caráter do papel da universidade como infraestrutura social. (Klinenberg, 2018; Latham, A., \& Layton, J, 2019) Buscamos aproximar o campus desses locais de confluência social, tornando possível ainda uma ressignificação positiva dos dispositivos sociotécnicos frequentemente associados aos prejuízos que causam à convivência social.

Outro ponto relevante que podemos levantar é a disponibilidade dos dados. Em um momento em que a interdição da universidade tornaria impossivel um estudo do seu espaço físico mais direto, a base de dados digital contendo padrões de uso e trajetórias semânticas dentro do campus foi um instrumento precioso, de rápida consulta e que não representou nenhuma despesa financeira além daquelas que consistem na própria manutenção da rede.

$\mathrm{Na}$ literatura se convencionou aproximar o emprego de tecnologias de big data e sensores abaixo da alcunha de Smart City, objeto ao qual alguns autores apresentam válidos contrapontos. Adam Greenfield é um dos críticos mais ativos neste sentido. Greenfield (2013) associa a noção de cidade inteligente à visão determinista de empresas que lucram e potencialmente podem lucrar ainda mais com a intensificação do fluxo global de bits através de um planeta repleto de cidades inteligentes. São lugares que, segundo o autor, têm uma natureza genérica, específica e fechada. Este último, principalmente deve-se ao fato das plataformas utilizadas pelas principais empresas que vendem sistemas de "cidades inteligentes" no mundo não possuírem código aberto.

Deve-se ter em conta também que os objetivos sociais das cidades e de seus espaços públicos, como o campus, e as forças que tradicionalmente atuam na consolidação das formas urbanas devem estar atrelados aos processos políticos e democráticos. Faz parte da intenção de se produzir esse tipo de conhecimento o interesse em permitir aos pesquisadores identificarem digitalmente os aspectos não digitais das cidades. Consideramos positivamente o peso de aproximarmos essas tecnologias do conhecimento técnico de arquitetos e urbanistas, possibilitando que a noção de Smart City seja pensada criticamente além dos procedimentos padrões das empresas que desenvolvem esses softwares e hardwares. Por isso também a relevância do uso de plataformas abertas e compartilháveis como as que aqui apresentamos. À medida em que aproximamos um campus urbano como o de UFSC da cidade que o circunda e até então é afastada simbólica e espacialmente da universidade, sobretudo reforçando suas características de espaço público, podemos vislumbrar um futuro de comunidade acadêmica e população da cidade menos afastados territorial e socialmente.

\section{AGRADECIMENTOS}

Agradecemos ao Conselho Nacional de Desenvolvimento Científico e Tecnológico (CNPq), à Coordenação de Aperfeiçoamento de Pessoal de Nível Superior (CAPES), ao Laboratório de Segurança em Computação (LabSEC UFSC) e à Superintendência de Governança Eletrônica e Tecnologia da Informação e Comunicação (SeTIC UFSC). 


\section{REFERÊNCIAS}

Baras, Karolina, e Adriano Moreira. 2010. "Anomaly detection in university campus WiFi zones". 8th IEEE International Conference on Pervasive Computing and Communications Workshops, 202-7. doi.org/10.1109/PERCOMW.2010.5470669

Bratton, B. H. (2016). The stack: On software and sovereignty. Cambridge, MA: MIT Press.

Brasil. (2018). Lei n. 13.079, de 14 de agosto de 2018. Lei Geral de Proteção de Dados Pessoais (LGPD). Diário Oficial da União.

Cots, M., \& Oliveira, R. (2019). Lei geral de proteção de dados pessoais: comentada. Revista dos Tribunais.

Greenfield, Adam. (2013) Against the smart city: A Pamphlet. Nova York, NY: Do Projects.

Klinenberg, E. (2018). Palaces for the people: How social infrastructure can help fight inequality, polarization, and the decline of civic life. Nova York, NY: Broadway Books.

Latham, A., \& Layton, J. (2019). Social infrastructure and the public life of cities: Studying urban sociality and public spaces. Geography Compass, 13(7), e12444. doi: doi.org/10.1111/gec3.12444

Louail, Thomas, Maxime Lenormand, Oliva G Cantu Ros, Miguel Picornell, Ricardo Herranz, Enrique Frias-Martinez, José J. Ramasco, e Marc Barthelemy. (2014). From mobile phone data to the spatial structure of cities. Scientific reports $4: 5276$. doi: doi.org/10.1038/srep05276

Loukissas, Y. A. (2016). A place for Big Data: Close and distant readings of accessions data from the Arnold Arboretum. Big Data \& Society, 3(2), $2053951716661365 . \quad$ doi: doi.org/10.1177/2053951716661365

Mangrich, C. P., Pavan, L. H., Gomes, F., Oliveira, L., Kos, J., \& Martina, J. E. (2019). Campus regenerative design supported by university Wi-Fi connections. International Journal of Architectural Computing, 17(2), 206-219. doi: doi.org/10.1177\%2F1478077119849682
Mulholland, C. S. (2018). Dados pessoais sensíveis e a tutela de Direitos Fundamentais: uma análise à luz da Lei geral de Proteção de Dados (Lei 13.709/18). Revista de Direitos e Garantias Fundamentais, 19(3), 159-180. doi: doi.org/10.18759/rdgf.v19i3.1603

Naboulsi, Diala, Marco Fiore, Stephane Ribot, e Razvan Stanica. (2015). Large-scale mobile traffic analysis: a survey. IEEE Communications Surveys \& Tutorials 18 (1): 124-61. doi: doi.org/10.1109/COMST.2015.2491361

Ratti, C., Frenchman, D., Pulselli, R. M., \& Williams, S. (2006) Mobile landscapes: using location data from cell phones for urban analysis. Environment and planning B: Planning and design, 33(5), 727-748. doi: doi.org/10.1068/b32047

Yang, P. P., \& Yamagata, Y. (2020). Urban systems design: shaping smart cities by integrating urban design and systems science. In Urban Systems Design (pp. 1-22). Elsevier. doi: doi.org/10.1016/B978-0-12-816055-8.00001-4

Yoshida, T., Yamagata, Y., Chang, S., de Gooyert, V., Seya, H., Murakami, D. e Voulgaris, G. (2020). Spatial modeling and design of smart communities. In Urban Systems Design (pp. 199-255). Elsevier. doi: doi.org/10.1016/B978-0-12-816055$8.00007-5$

Universidade Federal de Santa Catarina, (2020). Câmara de Graduação da UFSC aprova proposta de Calendário Suplementar Excepcional. Obtido em: noticias.ufsc.br/2020/08/camara-de-graduacao-da-ufscaprova-proposta-de-calendario-suplementar-excepcional/

Yang, Y., Peng, F., Wang, R., Guan, K., Jiang, T., Xu, G., ... \& Chang, C. (2020). The deadly coronaviruses: The 2003 SARS pandemic and the 2020 novel coronavirus epidemic in China. Journal of autoimmunity, 102434. doi: www.doi.org/10.1016/j.jaut.2020.102434

Organização Mundial da Saúde. Coronavirus disease (COVID-19) advice for the public. Obtido em: who.int/emergencies/diseases/novel-coronavirus2019/advice-for-public

Organização Mundial da Saúde. WHO Director-General's opening remarks at the mission briefing on COVID-19. Obtido em: who.int/dg/speeches/detail/who-director-general-s-openingremarks-at-the-mission-briefing-on-covid-19 